\title{
Homogenization of material properties in additively manufactured structures
}

\author{
Xingchen Liu, Vadim Shapiro* \\ Spatial Automation Laboratory \\ University of Wisconsin - Madison
}

\begin{abstract}
Additive manufacturing transforms material into three-dimensional parts incrementally, layer by layer or path by path. Subject to the build direction and machine resolution, an additively manufactured part deviates from its design model in terms of both geometry and mechanical performance. In particular, the material inside the fabricated part often exhibits spatially varying material distribution (heterogeneity) and direction dependent behavior (anisotropy), indicating that the design model is no longer a suitable surrogate to consistently estimate the mechanical performance of the printed component.

We propose a new two-stage approach to modeling and estimating effective elastic properties of parts fabricated by fused deposition modeling (FDM) process. First, we construct an implicit representation of an effective mesoscale geometry-material model of the printed structure that captures the details of the particular process and published material information. This representation of mesoscale geometry and material of the printed structure is then homogenized at macro scale through a solution of an integral equation formulated using Green's function. We show that the integral equation can be converted into a system of linear equations that is symmetric and positive definite and can be solved efficiently using conjugate gradient method and Fourier transform. The computed homogenized properties are validated by both finite element method and experiment results. The proposed two-stage approach can be used to estimate other effective material properties in a variety of additive manufacturing processes, whenever a similar effective mesoscale geometry-material model can be constructed.
\end{abstract}

Keywords: Additive manufacturing, Fused deposition modeling, Printed model, Heterogeneous material, Homogenization

\section{Introduction}

\subsection{Motivation and goals}

Additive manufacturing (AM) represents a spectrum of technologies producing 3D parts incrementally, layer by layer or path by path. This distinctive feature gives AM numerous advantages over the traditional manufacturing techniques, such as the ability to fabricate parts with complex shapes and internal structures without a significant increase in cost or turnaround time. In many cases, a complex heterogeneous structure with less material may be both cheaper and faster to manufacture than a part with a simpler geometry and homogeneous material, such as a solid cube. This phenomenon is sometimes referred to the "complexity paradox".

Similarly to other manufacturing methods, the quality of additively manufactured parts is subject to the process limitations and machine imprecision. Various differences between designed and manufactured parts have been studied in the past, in terms of surface roughness [1, 2], dimensional accuracy $[3,4]$, and other manufacturability criteria $[5,6]$.

\footnotetext{
*Corresponding author

Email addresses: xingchen@wisc.edu (Xingchen Liu), vshapiro@engr.wisc.edu (Vadim Shapiro)
}

Experiments have also been conducted to estimate the material properties of the printed part. The test specimens are printed in shapes per material testing standards (e.g. ASTM D3039 [7]) with material deposition paths (roads) aligned along the axial, transverse or cross directions $[8,9]$.

In contrast to many traditional manufacturing processes, the material undergoes a fundamental phase transformation during the AM process, changing not only its geometry but also its mechanical properties. Processing plan and parameters in AM also play a more significant role in the final performance of the part - the same nominal part geometry manufactured with two different set of process plans will generally result in parts with very different properties. As a result, all AM processes lead to a heterogeneous and anisotropic distribution of material properties in the interior of the fabricated part, which is usually not represented and accounted for in the part's design model. In other words, the design model is no longer a suitable surrogate for the fabricated part. The accuracy of downstream applications, such as structural analysis, relies on the ability to model not only the manufactured part's geometry but also its material's mechanical properties.

The material properties may be estimated at least three distinct scales: material phase changes take place at the micro scale, allowing planning material deposition at the 


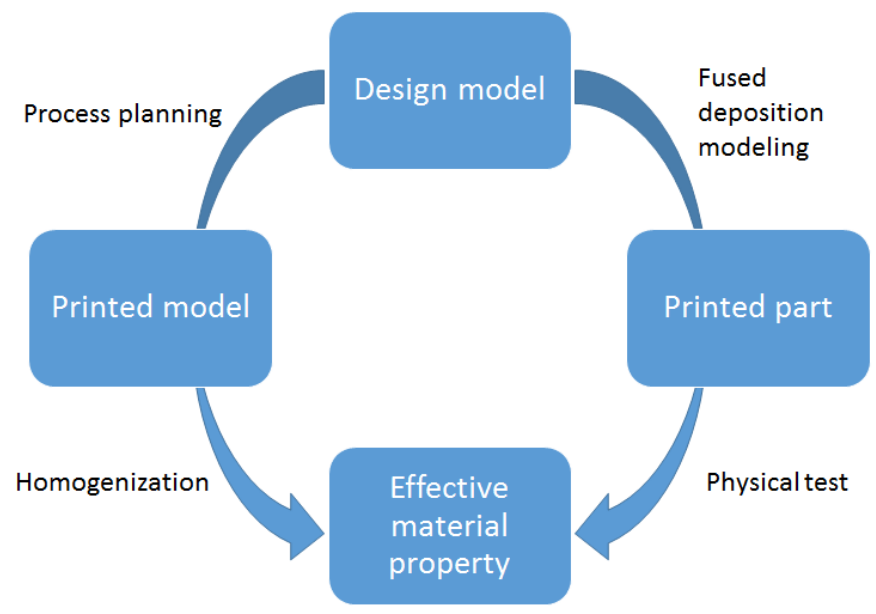

Figure 1: To model and estimate the effective material properties of parts made by FDM, the proposed approach includes two stages shown in the left branch: modeling of printed material and numerical homogenization. Direct simulation of the design model is likely to yield poor results due to the various inconsistencies between the design model and the printed part. The proposed method is verified with the physical testing result in [10] on the right branch in Section 5 .

(meso) scale of layers and paths, which are fused together to give effective mechanical properties of the manufactured part's (macro) scale. As AM is rapidly evolving from a technology to prototype products in the conceptual design stage into a manufacturing process for the end-use loadbearing functional components, it is imperative to develop a computational infrastructure that allows mechanical analysis to be performed directly on the manufactured part. Estimating such effective properties at the scale of the part's geometry is the goal of this paper.

\subsection{Contributions and outline}

Specifically, we propose a new approach to modeling and estimating the effective (macroscopic) material properties in the interior of the parts produced by the Fused Deposition Modeling (FDM) process using homogenization. Informally, homogenization replaces the known detailed geometry and multi-phase material properties at a finer scale by simpler 'effective' geometric domain and single-phase (solid) material properties at a coarser scale. The effective domain is usually a cuboid, and the effective material properties are estimated from average stress and strain relationship over the cuboid. Homogenization is challenging for AM parts for two reasons: (1) geometry and material properties may not be known at the micro and meso scales; and (2) homogenization requires significant computational resources.

The proposed approach deals with the two challenges in two stages (Figure 1): a modeling stage that generates a representation of (mesoscale) geometry and anisotropy of the material deposition in the interior of the part, followed by an efficient analysis stage homogenizing the generated $3 \mathrm{D}$-printed structure for its effective material properties.
The concept of homogenization is extensively used in both two stages.

In the modeling stage, given a manufacturing process plan in the form of G-code that describes the printer's toolpath, we construct an effective geometry-material model to represent the heterogeneous distribution and anisotropic material properties of the FDM printed structures. The construction combines an analytic model of geometry with experimentally measured material properties that are linked together by homogenization assumed in the measurement procedure. We also describe an implicit representation of this mesoscale effective geometry-material model that supports efficient queries and can be evaluated on demand for further processing. This is the first contribution of the paper detailed in Section 3.

In the second stage, described in Section 4, the mesoscale geometry-material model of the printed structure is homogenized to obtain the effective (macro-scale) material elasticity tensor. We adopt the Green's function method that is often used in the studies of random heterogeneous materials. We convert the formulated integral equation into a system of linear equations and show that the linear system is symmetric and positive definite with properly chosen reference material. This is our second contribution, which gives a formal basis for using efficient homogenization techniques. The symmetric and positive definite linear system is solved by the conjugate gradient (CG) method. The matrix - vector multiplication required by $\mathrm{CG}$ is equivalent to the convolution between Green's operator and the vector of polarized stresses, and thus can be evaluated efficiently through Fourier transform.

To validate our results, in Section 5, we show that the computed results are consistent with those obtained by a more traditional (but an order of magnitude slower) homogenization method based on finite element method. We also apply the complete two-stage modeling-homogenization approach to models of printed material samples and show that predicted effective material properties are in agreement with the physical tests performed on the same structures.

\section{Background and related work}

\subsection{Fused deposition modeling}

FDM is a widely used AM process that produces parts with significant material anisotropy and heterogeneity that cannot be neglected. Parts built by FDM differ noticeably from their design models due to many factors, including stair - stepping on the surface of the part, the rounding of sharp corners, air gaps and the use of infill patterns to save the printing material and printing time, impacting the mechanical performance of the part.

To manufacture by FDM, the design (solid) model is first converted to a stereolithography (STL) file, which represents the solid part by a triangle tessellation of its boundary. The STL model is subsequently sent to a process planning software (e.g. Slic3r [11]) that generates the 
printer's head toolpath together with the printing process specifications, such as build direction, nozzle diameter, and infill percentage. As the printer's head moves, a molten filament is extruded through a heated nozzle. For each layer, the nozzle moves following a piece-wise linear path horizontally. The material extruded along each line segment is commonly referred as a 'road.' After each deposition, the road solidifies and bonds with adjacent roads in both current and previously deposited layers. After the whole layer is deposited, either the nozzle or the printing plate shifts vertically to print the next layer.

Much research has been dedicated to model the geometric differences between the design model and printed part; a comprehensive survey is beyond the scope of this paper. For example, elliptical model of the road cross-section is proposed in [12] to analyze surface roughness distribution according to changes in the angle between the surface and the build direction. Manufacturability of the designed part is examined in [5] and [13] using morphological operations with voxel representation and polygonal offsets respectively. Manufacturability analysis identifies hard to print features such as bridges, spikes, and holes. More recently, Nelaturi and Shapiro [6] model the printed shape as the accumulation of material deposited at each translated print head position, which is mathematically represented as the convolution between smallest printing volume by the moving head and the nominal geometry. The model also captures process uncertainty by introducing probability density functions into the convolution. Because the process plan is represented as a stack of slices, only geometric differences over the boundary of the part are reflected in this formulation. A more detailed model of the process-planned geometry may be modeled by the actual geometry of the roads, with each road represented by a sweep (convolution) of the smallest printing volume over the actual toolpath [14].

The studies of the mechanical properties of the printed material rely heavily on the experiments. For ABS plastic, experimental results show that the tensile strength along the road is about $75 \%$ weaker than the raw material [8]. The same study also found that the bonds between roads are much inferior to the raw material (20\% to $50 \%$, depending on the size of the air gap). This observation has been repeatedly confirmed by later experiments $[15,16,9]$. In particular, FDM parts with different build directions are compared with injection molded monolithic part with the same geometry so that the material properties in the printed part can be compared with the raw material directly [9]. The anisotropic mechanical properties of the printed part by different AM processes are reviewed in [17].

Our mesoscale effective geometry-material model combines an implicit sweep-based geometric model of the material deposition process with measured material properties. The combination is not trivial because, strictly speaking, the two models are incompatible. For example, as we explain in Section 3, the mechanical properties measured as an average over some domain of material sample do not hold for individual roads (solidified filament).

\subsection{Homogenization using Green's function}

Relating the macroscopic properties to the microscopic material structures is a long-standing problem. Informally, homogenization refers to the process of calculating the effective properties of a material structure $[18,19]$. One popular strategy for homogenizing a structure numerically is though Finite Element Method (FEM) where six independent periodic boundary conditions are imposed [20]. Multiple recent studies adopt FEM-based homogenization to design and optimize mesoscale structures for additive manufacturing. Without being exhaustive, a set of cubic patterns achieving a broad range of stiffness and Poisson's ratios is designed through a combinatorial search over topologies followed by shape optimization in [21]. A similar result through topology optimization is reported in [22]. By imposing manufacturing constraints, manufacturable material structures with negative Poisson's ratios are designed through topology optimization in [23].

An alternative approach to homogenization can be formulated using Green's function (see Appendix A). The development of the method dates backs to $1970 \mathrm{~s}$ $[24,25,26,27]$. A major computational difficulty in the formulated integral equation is computing the convolution of Green's function. Initially, the integral equation was used to predict effective properties or estimate their bounds based on the statistics of microscopic material structures [28]. It is only recently an efficient solution of the integral equation was proposed, allowing the application of the approach to the detailed models of material structures. Evaluating the convolution using Fourier transform was first proposed by Moulinec and Suquet [29]: by the convolution theorem, the convolution in real domain becomes multiplication in the frequency domain. Just as in FEMbased homogenization, the periodic boundary conditions are usually assumed for the material [19]. Fourier transform also assumes the periodicity of the function implicitly. The first attempt [29], often referred as the basic scheme, solves the problem through the fixed-point iteration which is equivalent to the Neumann series solution of the integral equation. In this approach, the number of iterations needed for convergence grows linearly with the contrast in material coefficients between different phases. ${ }^{1}$ As a result, the iteration may not converge for phases with infinite contrast, e.g. porous materials. It was later discovered that the lack of convergence is due to the Neumann series expansion rather than the formulation of the problem and will be corrected if other iterative linear solvers are applied [30]. The basic scheme inspired many follow-up results, mainly focusing on improving the convergence speed and the convergence with arbitrary phase contrast $[31,32,33,34]$. The Fourier transform of the problem was later shown to be mathematically

\footnotetext{
${ }^{1}$ Contrast refers to the magnitude of difference between the coefficients in the respective material tensors.
} 

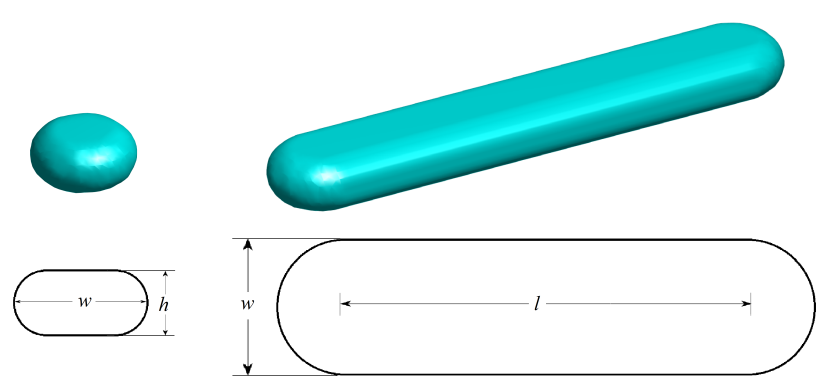

Figure 2: The shape of the minimum manufacturing volume and a single road. $w, l$, and $h$, represent the width, length of the road and layer height, respectively.

equivalent to Galerkin approximation with trigonometric polynomials [35]. The efficient homogenization technique described in Section 4 relies on the combination of FFT and conjugate gradient methods, exploring the fact that the resulting system of linear equation is symmetric and positive definite.

\section{Mesoscale FDM Geometry-Material model}

\subsection{Idealized geometry of printed structures}

Intuitively, we would like to simulate the FDM printing process as a deposition of filament along the specified toolpath. At any given instant, the printer's head deposits some minimum manufacturing volume (MMV), whose shape may be approximated in terms of simple quadratic and/or superelliptic primitives with dimensions determined by the road width and layer height (see Figure 2 left). The shape of the road may be represented as the sweep of MMV along the toolpath specified by the instructions in the G-code, as shown on the right. Sweeping MMV over all toolpaths in the G-code yields a first approximation of geometry discretized by the printing process. Figure 3 shows the design model, toolpath, and the reconstructed printed shape of a two-dimensional infill pattern generated through Customizer by MakerBot [36]. Such an idealized geometry model was proposed in [14] and can be used to reason about stair-stepping, surface roughness, air gaps, and other small geometric differences between the designed and printed model.

We will adopt this idealized geometric model as a first approximation of the mesoscale geometry-material model, based on the assumption that most of the differences between the mechanical performance of the designed and printed structures originate in the process planning stage. This is reasonable because the air gaps and the bonding interface between the roads, which are modeled by the idealized geometry model, impact the mechanical performance of the printed structure far more significantly than dimension inaccuracy and printing imperfections due to gravity and random events such as inconsistent polymer flow. Global deformations such as warping due to thermally induced stress are not modeled since they cannot be predicted without first solving the effective material problem under consideration in this paper. Another limitation of the idealized geometry model is that the union of the roads is not volume conservative. The model will underestimate the total volume of the base material when the distance between the parallel toolpath is smaller than the road width. We expect that such problem is less likely to happen with a proper toolpath.

\subsection{Mesoscale material properties}

A standard method for modeling a multi-phase material structure is to partition the overall domain into regions with distinct but known material properties. Thus, idealized geometry model distinguishes the space occupied the roads from the voids. However, the elastic properties of the roads themselves vary significantly over the roads and are not generally known. Firstly, it is very difficult to predict the properties of the solidified road base material that is remelted from the filament and extruded through the nozzle during the deposition process. Furthermore, the elasticity tensor of the filament itself is likely to be incomplete since only the stiffness in the direction of filament's extrusion can be tested in most scenarios. Secondly, the stiffness of the deposited material changes significantly in the areas where adjacent roads are bonded together and is also difficult to predict. The quality of the bonds between the roads depend not only on the neck (contact area) formed between the adjacent roads, but also the molecular diffusion and randomization at the interface [37]. Heat transfer and thermal history models, such as the ones proposed in [38, 37], are required to predict the strength and stiffness of the bond. The compound complexity of these physical phenomena casts serious doubts on the feasibility of being able to predict material properties of the idealized geometry model either computationally or experimentally.

In contrast, the measured material properties published by various manufacturers estimate not material properties of the constituent phases, but the effective material properties of the specimen formed by uniform patterns of parallel roads (Figure 4) (top). These measurements determine the compounded effects of the stiffness of the roads, air gaps and the bonding between the roads; the properties are averaged over an effective domain, shown as the blue rectangular region in Figure 4 (middle) where they are assumed to be constant. Clearly, these measured properties cannot be applied directly to the idealized geometry model constructed from a number of roads with arbitrary orientation and spacing.

\subsection{Effective geometry - material model}

It should now be clear that the effective geometry and material models of the FDM structures are interdependent and cannot be treated separately. The difficulty is that this interdependence is implicit in the (inconsistent) assumptions underlying the geometry model and material measurement procedures, but it cannot be used explicitly to 


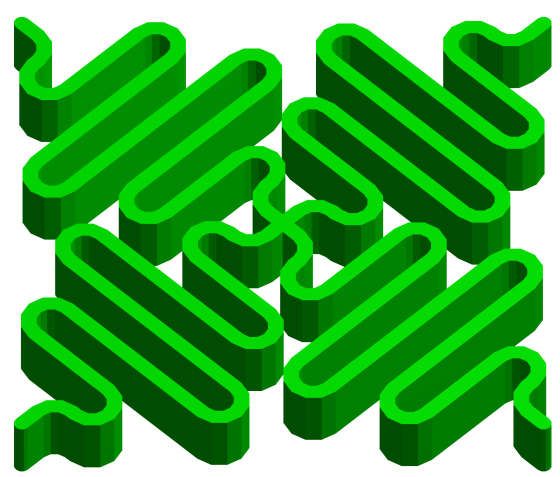

(a) Design model represented by triangle tessellation.

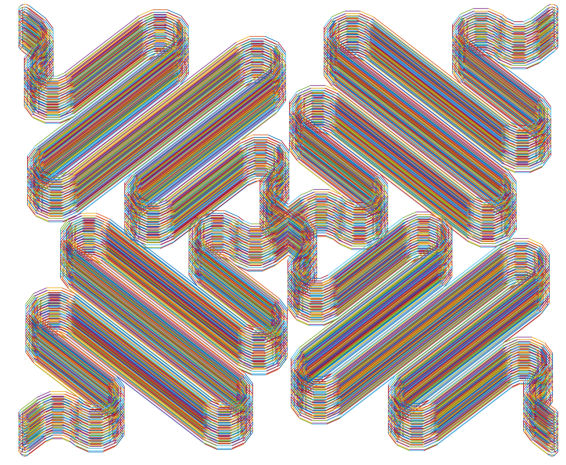

(b) Toolpath as a collection of line segments.

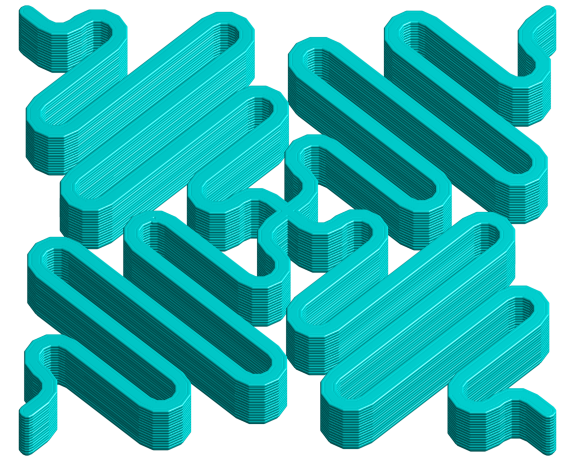

(c) Printed model reconstructed from the toolpath (b) with road width $w=0.3$.

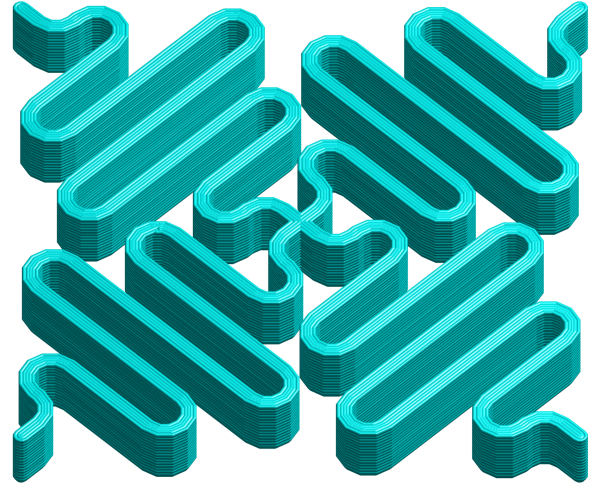

(d) Reconstructed printed model with $w=0.25$.

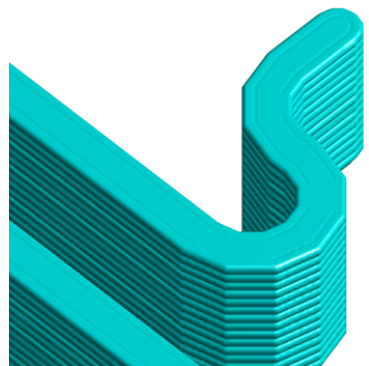

(e) Detailed view of (c).

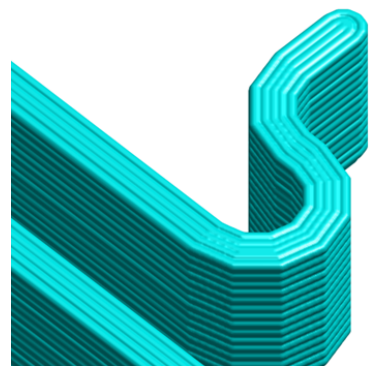

(f) Detailed view of (d).

Figure 3: Printed geometry model for a two-dimensional infill pattern.

express either model in terms of the other. Hence, we propose to simultaneously modify both models of geometry and of material, in order to bring them into the agreement and construct a consistent effective geometry-material model.

First, the idealized road shown in Figure 2 is reinterpreted with a rectangular cross-section shown at the bottom of Figure 4. This reinterpretation is consistent with the material measurement process that assumes the effective rectangular cross-section of the specimen shown in Figure 4 does not have any voids. Secondly, because the measurement specimen is a periodic arrangement of parallel roads, the measurement procedure implies that the measured material properties remain constant and are identical on the cross-section of each individual road. This means that the measured anisotropic material properties can now be assigned based on the reinterpreted geometry and the direction of the road, while eliminating the need to model air gaps and bonding stiffness. ${ }^{2}$

Next, we transform the measured material properties to be consistent with the direction of individual roads. The material properties are measured in a coordinate system with $z$ axis pointing in the build direction and $x$ axis aligned

\footnotetext{
2 Printing defects such as imperfect bonding must be treated separately with thermal history models, which is outside the scope of current discussion.
}

with the (stiffest) direction of the roads in the specimen. The measurement results are generally represented by the following transverse isotropic compliance tensor:

$$
S=\left[\begin{array}{cccccc}
\frac{1}{E_{x}} & -\frac{\nu_{x p}}{E_{x}} & -\frac{\nu_{x p}}{E_{x}} & 0 & 0 & 0 \\
-\frac{\nu_{x p}}{E_{x}} & \frac{1}{E_{p}} & -\frac{\nu_{p}}{E_{p}} & 0 & 0 & 0 \\
-\frac{\nu_{x p}}{E_{x}} & -\frac{\nu_{p}}{E_{p}} & \frac{1}{E_{p}} & 0 & 0 & 0 \\
0 & 0 & 0 & \frac{1+\nu_{p}}{E_{p}} & 0 & 0 \\
0 & 0 & 0 & 0 & \frac{1}{2 G_{x p}} & 0 \\
0 & 0 & 0 & 0 & 0 & \frac{1}{2 G_{x p}}
\end{array}\right]
$$

where $E_{p}, \nu_{p}$ and $E_{x}, \nu_{x}$ are Young's moduli and Poisson ratios in the $y-z$ symmetry plane and $x$-direction, respectively. $G_{x p}$ is the shear modulus in the $x$-direction. The elasticity tensor $C$ is calculated as the inverse of the compliance tensor $S$. Note that $y-z$ is the symmetry plane, which is different from the usual convention that $x-y$ is the symmetry plane.

The roads can be printed along any direction within the layer while remaining perpendicular to the build direction. To align the $x$ axis of measurement with the road direction, this fourth-order elasticity tensor must be rotated within the layer [39]:

$$
C_{m n o p}^{\prime}=R_{m i} R_{n j} R_{o k} R_{p l} C_{i j k l}
$$



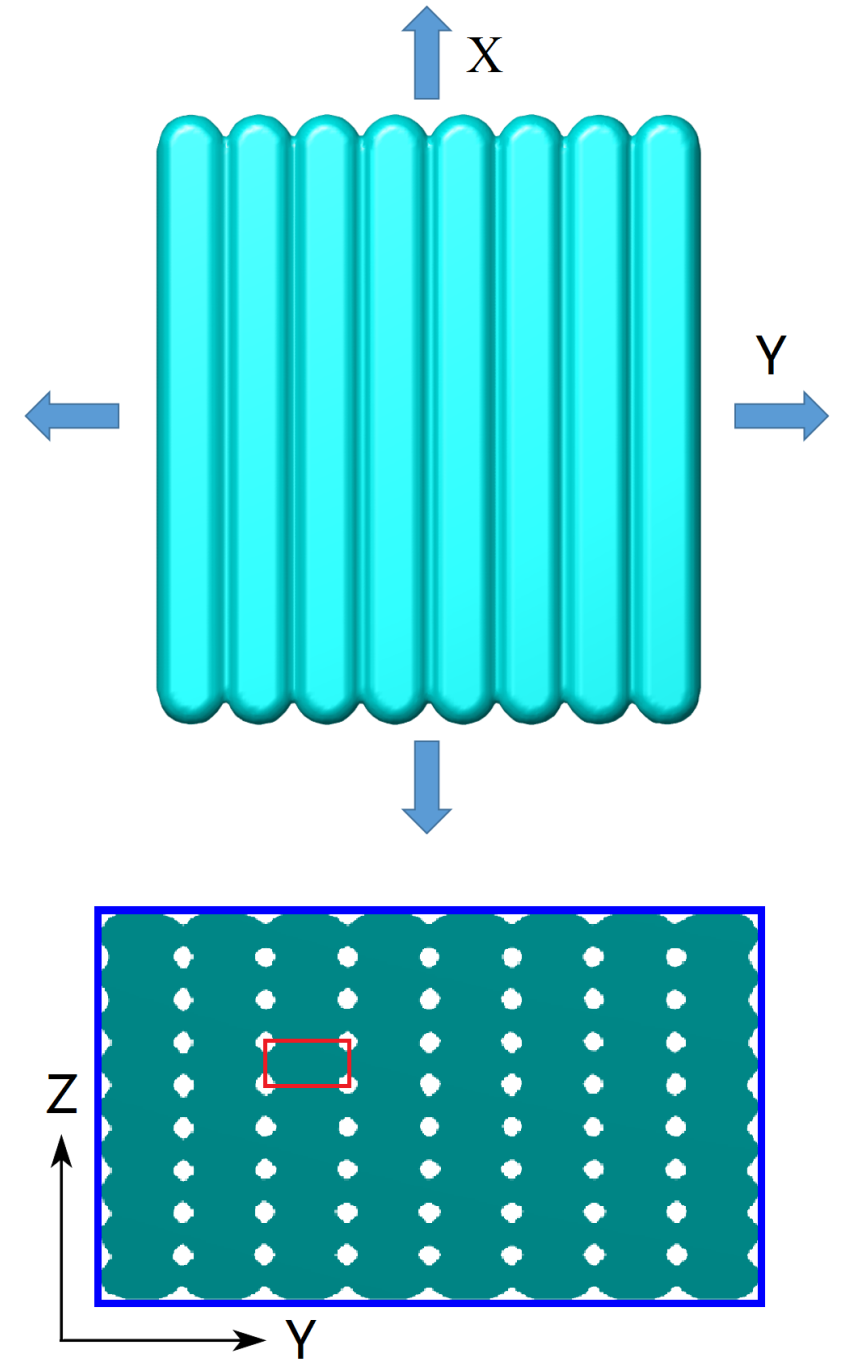

Figure 4: The material properties of printed structure are measured on a specimen formed by a uniform pattern of parallel roads. Top: tensile tests are performed along $\mathrm{X}$ and $\mathrm{Y}$ directions. Middle: the cross-section of the specimen. The blue rectangle indicates an effective domain of the measurement. The red rectangle indicates an effective cross-section of a road that is compatible with the assumptions in the measurement. Bottom: reinterpreted geometry of a single road.

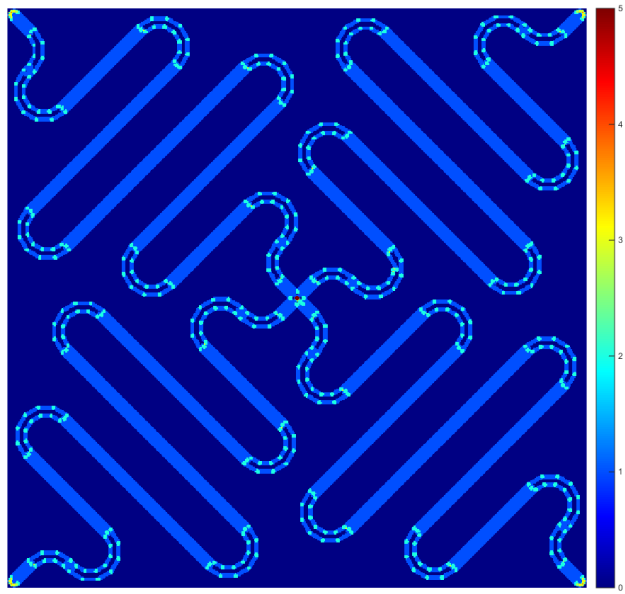

(a) Discretized first layer. The number of roads each query point belongs to is color coded. The averaged material property is returned if the query point belongs to more than one road.

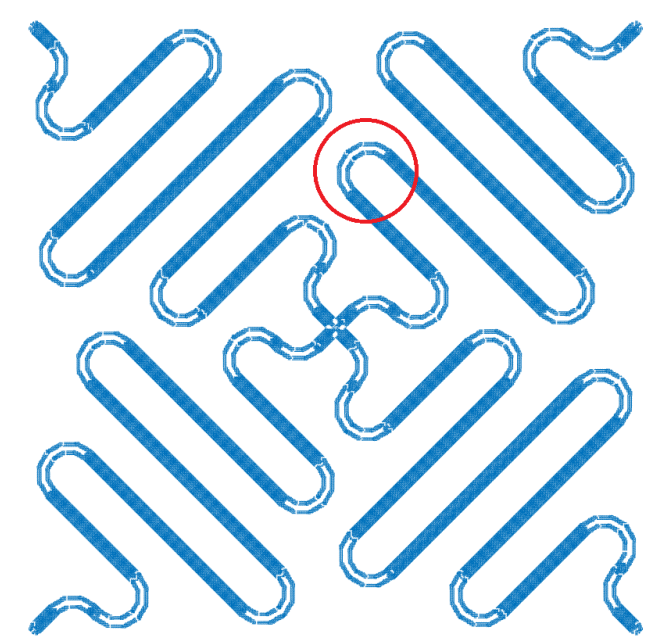

(b) The stiffest direction of material tensor for each query point.

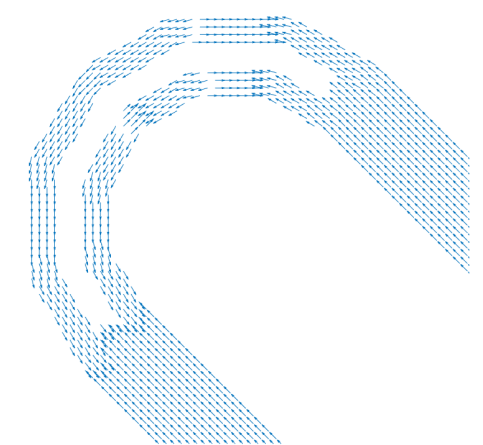

(c) The zoomed in view of (b).

Figure 5: Material distribution of printed model for a two-dimensional infill pattern. 
where $R_{i j}$ is the ij-th component of $R$ :

$$
R=\left[\begin{array}{ccc}
\cos \theta & -\sin \theta & 0 \\
\sin \theta & \cos \theta & 0 \\
0 & 0 & 1
\end{array}\right]
$$

and $\theta$ is the angle between the $x$ axis and the road direction. Figure 5 shows the result of aligning the material tensor with the directions of the roads within a single layer. Here, the stiffest direction of the elasticity tensor is sampled at different locations and is plotted as a vector field.

One final modification of material model is required to account for areas where multiple roads overlap. In such cases, the elasticity tensor is simply averaged over all overlapping roads. For example, if the overlap is between two parallel roads, the elasticity tensor remains the same as in both roads. If two roads form a T-section, the returned elasticity tensor becomes isotropic in the plane of the layer after averaging from the two roads.

\subsection{Implementation via implicit representation}

The mesoscale geometry-material model is conveniently represented by extending the implicit sweep-based representation of the idealized printed geometry. The point membership of a given query point against such a representation is determined by its point membership against the MMV centered at its nearest point on the toolpath.

The same implicit representation is adapted and reinterpreted to be consistent with the proposed effective geometry - material model. A transformed material tensor is associated with every road. When a material tensor is queried at a point, both the point and the relevant roads are simply projected onto the horizontal $x-y$ plane of the layer (perpendicular to the build direction). The two-dimensional point membership classification against the projected road effectively eliminates the air gaps between the adjacent bonded roads, while other voids within the layers can still be recovered. This implicit representation of the effective mesoscale geometry-material model is sufficient for many downstream applications; in particular, it forms the input for the macro scale homogenization stage discussed in the next section.

\section{Homogenization via Green's function}

In principle, the mesoscale model developed in Section 3 may be used directly for performing mechanical analysis on an FDM printed part. However, this may not be desirable for at least two reasons. Discretization of the domain at the scale finer than the road geometry will result in an excessively large simulation model. Secondly, as we already explained, application of the measured material properties to effective mesoscale geometry is a form of homogenization. As such, this mesoscale model shares the usual accuracy limitations of homogenization in the vicinity of (relatively) small features and boundaries. ${ }^{3}$

For these reasons, below we further homogenize the constructed mesoscale geometry-material model to estimate the macroscopic material properties in the interior of an FDM printed structure. Because local forces and displacement are further averaged over an effective macroscopic domain, it is reasonable to expect that the accuracy limitations of the mesoscale model will have limited or no effect on results of this homogenization.

\subsection{The governing integral equation}

We rely on the formulation developed in [25, 27] and summarize its core result below. The effective elasticity tensor $C^{\text {eff }}$ of a heterogeneous material can be defined as:

$$
\bar{\sigma}=C^{\mathrm{eff}} \bar{\epsilon}
$$

where $\bar{\epsilon}$ and $\bar{\sigma}$ are the mean value of strain $\epsilon$ and stress $\sigma$ in the material sample. Note that the effective tensor is almost never the volume average of its constituent materials. In fact, the volume average serves as an upper bound of the effective properties and is almost never achieved in practice.

Due to the symmetry of the strain and stress tensor, a set of six independent experiments is required for measuring $C^{\text {eff }}$. For a heterogeneous material with elasticity tensor $C$ subject to the displacement boundary condition, the displacement $u$, stress $\sigma$, strain $\epsilon$, and body force $b$ must conform to the following equilibrium equations:

$$
\begin{gathered}
\operatorname{div} \sigma+b=0, \quad x \in \Omega \\
\sigma=C \epsilon, \quad \epsilon=\frac{1}{2}\left[\nabla u+(\nabla u)^{T}\right], \\
u(x)=u^{0}(x), \quad x \in \partial \Omega .
\end{gathered}
$$

Given a homogeneous reference comparison material with the constant elasticity $C^{0}$, the stress polarization tensor $\tau$ may be defined:

$$
\tau=\sigma-C^{0} \epsilon=\left(C-C^{0}\right) \epsilon .
$$

Substituting Equation (6) into (5) leads to the solution of $\epsilon$ (see derivation in Appendix B), also known as the Lippmann-Schwinger equation:

$$
\begin{gathered}
\epsilon(x)=-\int_{\Omega} \Gamma\left(x, x^{\prime}\right) \delta C\left(x^{\prime}\right) \epsilon\left(x^{\prime}\right) \mathrm{d} x^{\prime}+\epsilon^{0}(x) \\
\Gamma_{i j p q}\left(x, x^{\prime}\right)=\left.\frac{\partial^{2} G_{i p}\left(x, x^{\prime}\right)}{\partial x_{j} \partial x_{q}^{\prime}}\right|_{(i j),(p q)}
\end{gathered}
$$

where the bracketed subscripts imply symmetries on $(i j)$ and $(p q), \epsilon^{0}$ is a constant strain field resulting from the

\footnotetext{
${ }^{3}$ Roughly speaking, effective properties are used when the feature size is at least an order of magnitude larger than the size of the effective geometric domain.
} 
prescribed $u^{0}$, and $\delta C$ represents the variations in elasticity from the reference material: $\delta C=C-C^{0}$. $G$ is the Green's function of an infinite size material:

$$
\left[C_{i j k l}(\boldsymbol{x}) G_{k p, l}\left(\boldsymbol{x}, \boldsymbol{x}^{\prime}\right)\right]_{, j}+\delta_{i p} \delta\left(\boldsymbol{x}-\boldsymbol{x}^{\prime}\right)=0 .
$$

The close form expressions of $\Gamma$ and $G$ is explicitly known in the frequency domain, as summarized in Appendix A.

The solution of Equation (7) is the strain tensor distribution $\epsilon(x)$ within the material domain. The stress tensor field is subsequently computed as the point-wise multiplication of the modeled material elasticity tensor field by the strain tensor distribution: $\sigma(x)=C(x) \epsilon(x)$. Our implementation follows Mandel's notation, where the $2 n d$-rank strain and stress tensors are mapped to six-dimensional vectors and the $4 t h$-rank elasticity and compliance tensors are mapped to $6 \times 6$ matrices. After repeating this process six times independently, the effective elasticity tensor can be computed as:

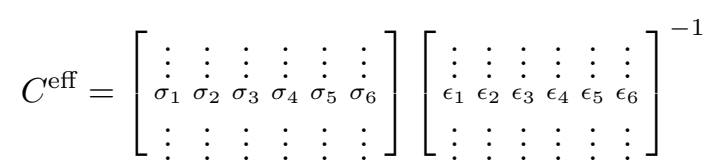

If the input strain vector is of unit length and parallel to the axes, the strain matrix in Equation (9) will be an identity matrix so that the inversion is not required. This is because the averaged strain always equals to the prescribed constant strain per Equation (16). This identity can also be used to verify the implementation of the algorithm.

Equation (7) is a Fredholm integral equation of the second type, whose classical solution requires Neumann series expansion [40]. First proposed by Moulinec and Suquet [29], the integral term in Equation (7) is efficiently evaluated through Fourier transform. In their work, a fixedpoint iteration following the Neumann series expansion is proposed:

$$
\epsilon^{i+1}(x)=\epsilon^{i}(x)-\mathcal{F}^{-1}\left[\hat{\Gamma} \mathcal{F}\left(\delta C \epsilon^{i}(x)\right)\right]
$$

where $\mathcal{F}$ and $\mathcal{F}^{-1}$ represents the forward and inverse Fourier transform and $\hat{\Gamma}$ is the Green's operator in the frequency domain. As reviewed in Section 2, this method suffers slow convergence when the contrast of material properties increases, which is undesirable for our application as the phase contrast goes to infinity with the existence of air gaps.

\subsection{Solution as a linear system}

We now describe a method to solve the Equation (7) by converting it into a system of linear equations. Though the integration kernel $\Gamma$ in Equation (7) is inseparable in the continuous domain, it can be separated approximately with the following piece-wise linear approximation:

$$
\begin{gathered}
\Gamma\left(x, x^{\prime}\right) \delta C\left(x^{\prime}\right) \approx \sum_{j=1}^{N} u_{j}(x) v_{j}\left(x^{\prime}\right), \\
u_{j}(x)=\Gamma(x, j) \delta C(j), \quad v_{j}\left(x^{\prime}\right)=\left\{\begin{array}{ll}
1, & \text { if } x^{\prime}=j \\
0, & \text { if } x^{\prime} \neq j
\end{array} .\right.
\end{gathered}
$$

The following linear system can be derived from Equation (7) (see Appendix C for details):

$$
\left[\delta_{i j}+\sum_{j=1}^{N} \Gamma_{i j} \delta C_{j}\right] \epsilon_{j}=\epsilon_{i}^{0} .
$$

Or in matrix form:

$$
\begin{aligned}
& A \epsilon=\epsilon^{0}, \\
& A=\left[\begin{array}{c:c:c:c}
I+\Gamma_{11} \delta C_{1} & \Gamma_{12} \delta C_{2} & \ldots & \Gamma_{1 N} \delta C_{N} \\
\hdashline \Gamma_{21} \delta \bar{C}_{1} & I+\Gamma_{22} \delta C_{2} & \cdots & \Gamma_{2 N} \delta \bar{C}_{N} \\
\hdashline \vdots & \vdots & \ddots & \vdots \\
\hdashline \Gamma_{N 1} \delta \bar{C}_{1} & \bar{\Gamma}_{N 2} \delta \bar{C}_{2} & \cdots & I+\Gamma_{N N} \delta \bar{C}_{N}
\end{array}\right]
\end{aligned}
$$

where $\Gamma_{i j}=\Gamma_{i-j}=\mathcal{F}^{-1}(\hat{\Gamma}(\xi))$. Note that every block in $A$ is a $6 \times 6$ matrix representing a 4 th rank tensor. Apparently, $A$ is not symmetric in general. However, by letting

$$
\begin{aligned}
B & =A \delta C^{-1} \\
& =\left[\begin{array}{c:c:c:c}
\delta C_{1}^{-1}+\Gamma_{11} & \Gamma_{12} & \ldots & \Gamma_{1 N} \\
\hdashline \Gamma_{21} & \delta C_{2}^{-}+\Gamma_{22} & \cdots & \Gamma_{2 N} \ldots \\
\hdashline \vdots & \vdots & \ddots & \vdots \\
\hdashline \Gamma_{N 1} & \Gamma_{N 2} & \cdots & \delta C_{N}^{-I^{2}}+\Gamma_{N N}
\end{array}\right],
\end{aligned}
$$

the linear system transforms into:

$$
B \delta C\left(\delta C^{-1} \tau\right)=\epsilon^{0} .
$$

$\delta C$ can be seen as the right preconditioner for the linear system with $\tau$ as the unknown. Since only $\epsilon$ is required for homogenization, we just need to solve the preconditioned system $B \delta C \epsilon=\epsilon^{0}$. $\Gamma$ is symmetric in that $\hat{\Gamma}$ is an even function by definition (Equation A.15). Given $\delta C$ is also symmetric as a elasticity tensor, $B$ is symmetric.

In order to solve this preconditioned linear system by the conjugate gradient (CG) method, $B$ also needs to be positive definite [41]. It has been proved that the definiteness of $B$ and $\delta C$ are consistent [42]. Here we give an intuitive explanation of the proof. First, $\Gamma$ is positive definite because it has positive diagonal entries and is diagonally dominant as $\Gamma$ decays rapidly with the increasing distance between $x$ and $x^{\prime}$. When $C_{0}$ is smaller than all the phases in $\Omega, \delta C$ is also positive definite. $B$ is therefore positive definite. When $\delta C$ is negative definite, the proof is more involved and is included in Appendix D. In the case when both $B$ and $\delta C$ are negative definite, $B \delta C$ becomes positive definite as both $B$ and $\delta C$ are symmetric.

Given the usually large $N$, constructing $A$ can be prohibitively expensive. Fortunately, for every iteration, CG only needs the results of $A \epsilon$ for any vector $\epsilon$ and there is no need to represent $A$ explicitly. Since $A \epsilon=\epsilon+\Gamma *(\delta C \epsilon)$, where $*$ represents convolution, the vector is efficiently computed in the frequency domain. Note that the combination of Fourier transform and CG has been explored before in [34], despite the authors' belief that the linear system is not symmetric. Our analysis of the integral equation and 
explicit derivation of the corresponding linear system above justifies the use of CG even though the corresponding linear system appears to be non-symmetric.

\section{Experiment results}

To demonstrate the effectiveness of the proposed approach, we compare the computed homogenization results with those predicted by the finite element method, and validate the predicted effective macro scale material properties by the known results of physical tests.

\subsection{Comparison with finite element method}

A voxelized finite element mesh of the mesoscale geometrymaterial printed model is created by every query point within the effective material domain as a linear element with 8 nodes. The output of FEA analysis is the nodal displacement field. The strain field can be derived by differentiating the displacement field numerically. Numerical differentiation is sensitive to the noise and may generate erroneous results. Instead, we compute the averaged strain and stress through the displacement and traction on the boundary of the material domain directly based on the following equations:

$$
\begin{aligned}
\bar{\epsilon}_{i j} & :=\frac{1}{|\Omega|} \int_{\Omega} \epsilon_{i j}(x) \mathrm{d} x=\frac{1}{|\Omega|} \int_{\partial \Omega} \frac{1}{2}\left(u_{i} n_{j}+u_{j} n_{i}\right) \mathrm{d} S \\
\bar{\sigma}_{i j} & :=\frac{1}{|\Omega|} \int_{\Omega} \sigma_{i j}(x) \mathrm{d} x=\frac{1}{|\Omega|} \int_{\partial \Omega} \frac{1}{2}\left(t_{i} x_{j}+t_{j} x_{i}\right) \mathrm{d} S,
\end{aligned}
$$

where $\Omega$ is the material domain and $\partial \Omega$ is its boundary. Equation (16) follows immediately from the divergence theorem.

A fictitious isotropic material with Young's modulus $E=1 \mathrm{GPa}$ and Poisson's ratio $\nu=0.3$ is assigned to the $2 \mathrm{D}$ infill pattern shown in Figure 3. The input material elasticity tensor field has a resolution of $296 \times 296 \times 16$ and is generated from the printed mesoscale model proposed in Section 3. A soft material $(E=0.01 \mathrm{GPa})$ is assigned to the void phase as the stiffness matrix in FEM becomes ill-conditioned with elements of zero stiffness. The reference material for the proposed homogenization method has Young's modulus $E=1.5 \mathrm{GPa}$ and Poisson's ratio $\nu=0.3$ so that the linear system remains positive definite. The effective elasticity tensors $C^{\text {eff }}$ and $C^{\text {fem }}$ computed respectively by the proposed homogenization method and the FEM-based method are:

$$
\begin{aligned}
C^{\mathrm{eff}} & =\left[\begin{array}{cccccc}
0.0327 & 0.0150 & 0.0139 & 0 & 0 & 0 \\
0.0150 & 0.0327 & 0.0139 & 0 & 0 & 0 \\
0.0139 & 0.0139 & 0.3335 & 0 & 0 & 0 \\
0 & 0 & 0 & 0.0338 & 0 & 0 \\
0 & 0 & 0 & 0 & 0.0338 & 0 \\
0 & 0 & 0 & 0 & 0 & 0.0254
\end{array}\right] \\
C^{\mathrm{fem}} & =\left[\begin{array}{cccccc}
0.0330 & 0.0141 & 0.0132 & 0 & 0 & 0 \\
0.0138 & 0.0330 & 0.0129 & 0 & 0 & 0 \\
0.0151 & 0.0151 & 0.4234 & 0 & 0 & 0 \\
0 & 0 & 0 & 0.0227 & 0.0005 & 0 \\
0 & 0 & 0 & 0.0005 & 0.0227 & 0 \\
0 & 0 & 0 & 0 & 0 & 0.0235
\end{array}\right]
\end{aligned}
$$

The in-plane symmetry of the structure is reflected in the simulated result as $C_{11}^{\mathrm{eff}}=C_{22}^{\mathrm{eff}}$ and $C_{13}^{\mathrm{eff}}=C_{23}^{\mathrm{eff}}$. The homogenization also captures the in-plane spring-like behavior, which is about 10 times less stiff in the $\mathrm{x}$ and $\mathrm{y}$ directions than the $\mathrm{z}$ direction. We attribute the small difference between $C^{\text {eff }}$ and $C^{\text {fem }}$ to the different boundary conditions used. In FEM, a simple uniform displacement boundary condition is used since the Lagrange multipliers are required to apply periodic boundary condition in FEM [43]. Both the proposed homogenization method and FEM are implemented in MATLAB. To homogenize the example in Figure 3, homogenization using Green's function took less than 3 hours to converge while the FEM required more than 24 hours on an Intel i7 computer with 16 gigabytes memory.

\subsection{Comparison with physical tests}

We also compare the predicted homogenization results with the physically measured effective tensile modulus reported in [10]. The samples for the physical testing are shown in Figure 6. During the tensile test, only displacements in the central neck region are measured for the evaluation of the effective tensile modulus of specimen. For comparison with the results of physical testing, the neck regions were reconstructed from the G-code and homogenized by the proposed approach.

The filament used for the FDM printing is ABS plastic made by Airwolf3D with measured diameter $2.82 \mathrm{~mm}$ and tensile modulus $2.25 \mathrm{GPa}$. The layer height is explicitly known from the G-code. However, the road width still needs to be computed from the G-code based on the conservation of volume if such information is not explicitly given. We estimate that the road width is roughly $0.6 \mathrm{~mm}$. With the distance between parallel roads being $0.4 \mathrm{~mm}$, this results in an overfill that eliminates most of the air gaps in regions of solid infills, such as the contour regions close to the boundary the specimen. Based on this observation, the axial stiffness $E_{x}$ is estimated as almost identical to the stiffness of the filament and the transverse stiffness $E_{p}$ is about $80 \%$ to $90 \%$ of the filament stiffness. Based on the experiment results in [44], the Poisson's ratio $\nu_{x p}$ and $\nu_{p}$ in the transverse isotropic elasticity tensor (Equation 1) are both set to be 0.39 and $G_{x p}$ is set to be $1.6 \mathrm{GPa}$.

The homogenized effective elasticity tensor is not directly comparable to the tensile testing result. For example, the homogenized elasticity tensor of Infill B with $E_{x}=2.25$ $\mathrm{GPa}$ and $E_{p}=1.8 \mathrm{GPa}$ is

$$
\left[\begin{array}{cccccc}
2.4166 & 1.1825 & 1.0978 & -0.0000 & -0.0001 & 0.0003 \\
1.1826 & 2.2511 & 1.0561 & -0.0001 & -0.0000 & 0.0005 \\
1.0977 & 1.0559 & 2.0888 & -0.0001 & -0.0001 & 0.0001 \\
-0.0000 & -0.0001 & -0.0001 & 0.9174 & -0.0003 & -0.0000 \\
-0.0001 & -0.0000 & -0.0001 & -0.0003 & 0.9977 & -0.0000 \\
0.0003 & 0.0005 & 0.0001 & -0.0000 & -0.0000 & 1.2634
\end{array}\right]
$$

To compute the tensile modulus along the $\mathrm{x}, \mathrm{y}$, and $\mathrm{z}$ directions, we assume that the homogenized structures are orthotropic. In this particular set of examples, such assumption is reasonable given the normal and shear components in the homogenized effective elasticity tensor are essentially not coupled. The tensile modulus along the axial directions can be computed with the orthotropic compliance tensor 

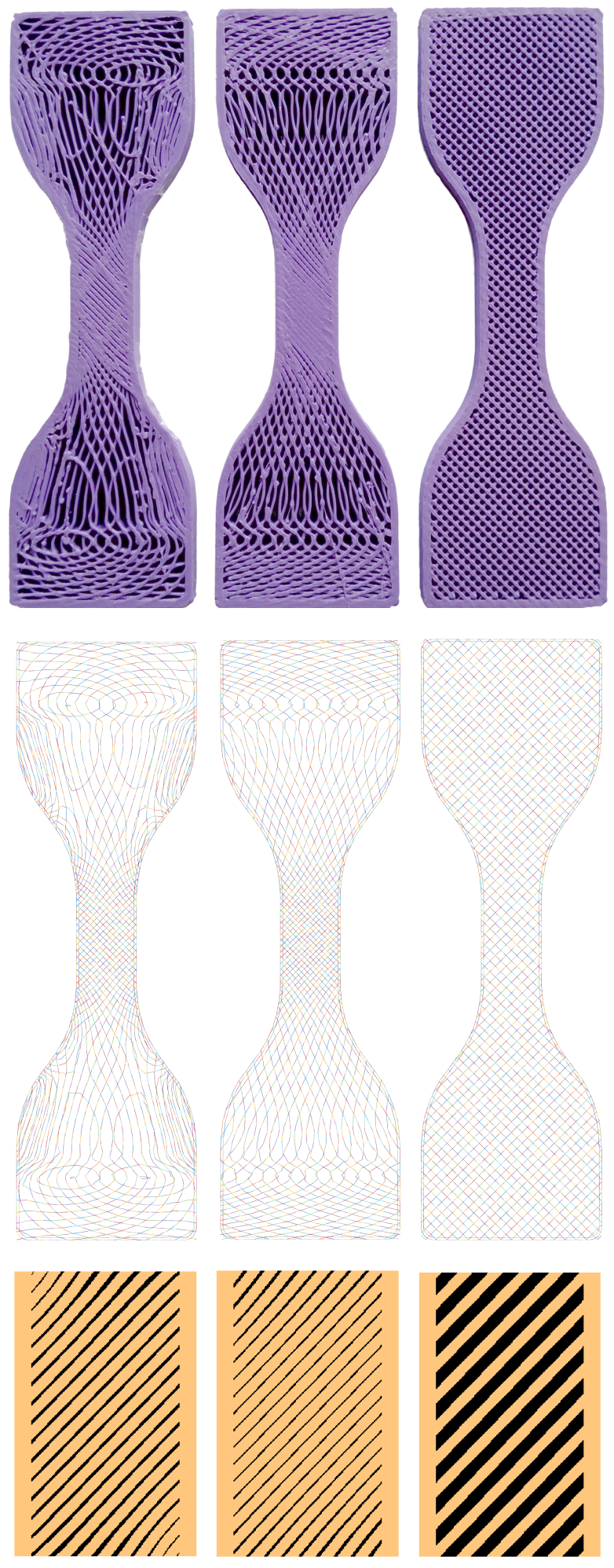

Infill $\mathrm{B}$
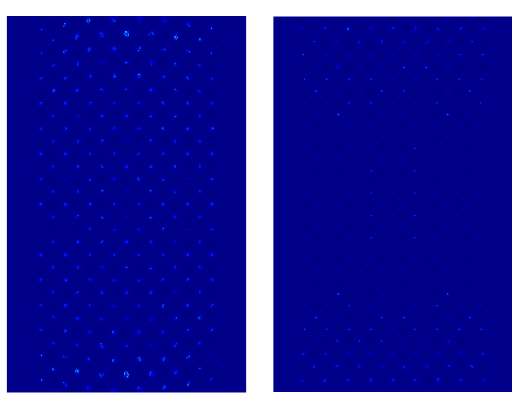

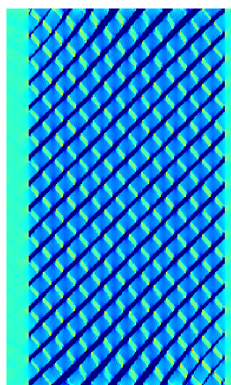

Infill A

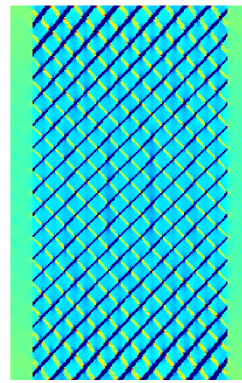

Infill B
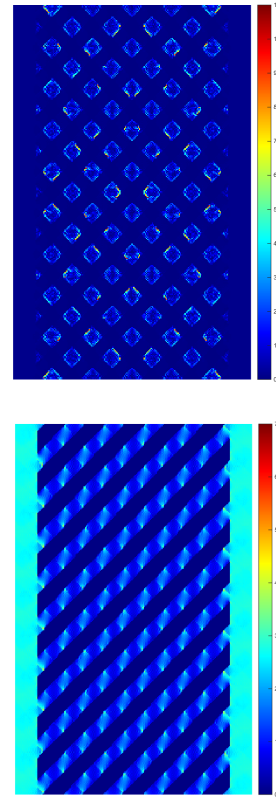

Infill linear
Figure 7: The strain and stress distributions in first layer of the homogenized regions with the displacement boundary condition along the vertical direction ( $\mathrm{x}$ axis) of the specimen. Top row: absolute value of $\epsilon_{x}$. Bottom row: absolute value of $\sigma_{x}$.

$S:$

$$
S=\left[\begin{array}{cccccc}
\frac{1}{E_{x}} & -\frac{\nu_{y x}}{E_{y}} & -\frac{\nu_{z x}}{E_{z}} & 0 & 0 & 0 \\
-\frac{\nu_{x y}}{E_{x}} & \frac{1}{E_{y}} & -\frac{\nu_{z y}}{E_{z}} & 0 & 0 & 0 \\
-\frac{\nu_{x z}}{E_{x}} & -\frac{\nu_{y z}}{E_{y}} & \frac{1}{E_{z}} & 0 & 0 & 0 \\
0 & 0 & 0 & \frac{1}{2 G_{y z}} & 0 & 0 \\
0 & 0 & 0 & 0 & \frac{1}{2 G_{z x}} & 0 \\
0 & 0 & 0 & 0 & 0 & \frac{1}{2 G_{x y}}
\end{array}\right] .
$$

It is clear that $E_{x}=\frac{1}{S_{11}}, E_{y}=\frac{1}{S_{22}}$, and $E_{z}=\frac{1}{S_{33}}$.

The measured and homogenized tensile moduli are reported in Table 1 with different stiffness in the transverse directions. The stress - strain curves for physical tests on different specimens are shown in Figure 8. We observe that the homogenized tensile moduli are smaller than the measured ones for Infill B and the linear infill. We believe this is due to the distance between the parallel roads being less than the road width. For example, the thickness of the vertical walls on the side of the infill pattern is reconstructed as $1 \mathrm{~mm}$ gives the $0.6 \mathrm{~mm}$ road width and 0.4 $\mathrm{mm}$ distance between the parallel toolpathes. However, the actual thickness of walls from the printed parts is about $1.2 \mathrm{~mm}$. The proposed printed model does not preserve the volume of the filament and underestimate the volume of the material in printed part with overfills. Since the G-code generator used in [10] is custom-built to study the effects of different infill patterns, the overfill is less likely to happen in most of the established process planning procedures. We also observed that the measured modulus of Infill A is around the midpoint of Infill B and linear infill. 
Table 1: The measured and homogenized tensile modulus by the proposed method. The first row shows the measured modulus (GPa) in [10]. The rest of the rows show the simulated modulus with $E_{x}=2.25 \mathrm{GPa}$ and different stiffness in the transverse direction. $E_{p}$ and $E_{x}$ are material coefficients of the transverse isotropic material tensor (Equation 1).

\begin{tabular}{cccc} 
& Infill A & Infill B & Linear \\
\hline Measured modulus [10] & 1.24 & 1.71 & 0.79 \\
$E_{p} / E_{x}=1$ & 1.4201 & 1.6769 & 0.7245 \\
$E_{p} / E_{x}=0.9$ & 1.3904 & 1.6396 & 0.7142 \\
$E_{p} / E_{x}=0.8$ & 1.3674 & 1.6103 & 0.7059 \\
$E_{p} / E_{x}=0.7$ & 1.3422 & 1.5784 & 0.6968
\end{tabular}

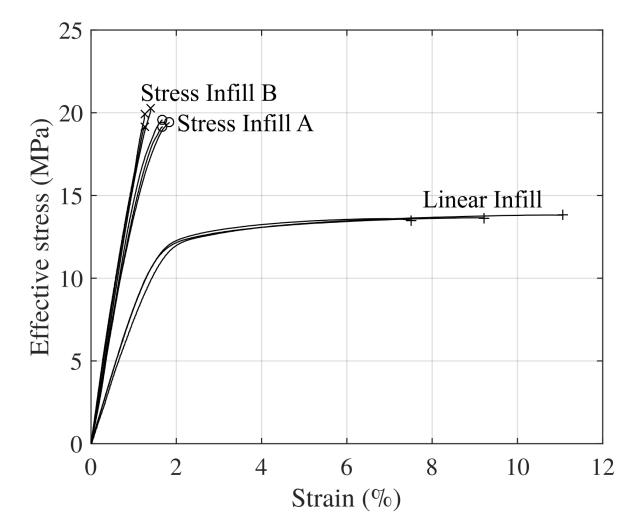

Figure 8: The stress - strain curve of physical testing result. Image courtesy of [10].

Even though our predicted modulus for infill A is higher than the measured result, it agrees with the stress - strain curve shown in Figure 8, in which the tensile moduli for Infill $\mathrm{A}$ and $\mathrm{B}$ are much closer.

\section{Conclusions}

\subsection{Summary and significance}

The main contribution of the paper is a new approach to predicting effective material properties of FDM printed structures. The approach is based on two novel ideas. First, given the manufacturing process plan and widely available material specifications, we formulated and constructed an implicit representation of an effective mesoscale geometrymaterial model of the printed structure that captures the heterogeneity and anisotropy resulting from the printing process. We then showed how this implicit representation of the mesoscale model may be queried and homogenized at macro scale in order to predict the effective material properties of the printed structure that accounts for build orientation, directional changes, infill patterns and other mesoscale details of 3D printing. We adopted and significantly improved the homogenization method using Green's function, showing that the corresponding linear system is symmetric and positive definite, and can be efficiently solved by the conjugate gradient method with matrix-vector multiplication evaluated in the frequency domain. The predicted effective material properties are in good agreement

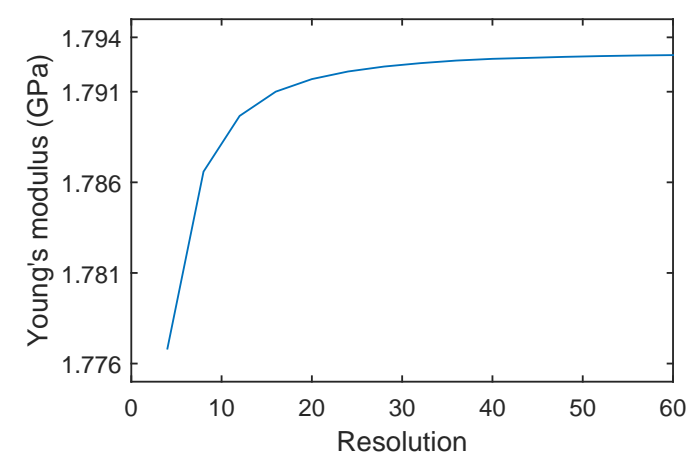

Figure 9: The change of effective Young's modulus with different sampling resolutions. Lower resolutions lead to the approximation errors on the Green's operator $\Gamma$.

with known experimental results and with the homogenization results predicted by the finite element method. The potent combination of implicit representations and queries handles the mesoscale complexity of FDM structures and is a further demonstration of the effectiveness of the querybased approach $[45,6]$ that avoids multiple representations and conversions of geometry and material models.

Our results point towards new opportunities in design, validation, and simulation of FDM printed structures. The ability to estimate the effective macro scale material properties implies that a heterogeneous material structure can be replaced with an equivalent homogeneous material, at least when the scale of the structural problem (the macro scale) and the scale of the material heterogeneity (the meso and micro scales) are well separated. The proposed approach is likely to be particularly effective for analyzing structures that are much larger than the printed road width and layer height. In such situations, the estimated effective material properties may be used for subsequent mechanical simulations, for example to predict warping, global deformations, and other performance and/or manufacturability characteristics. Different process plans may now be compared and optimized with respect to the effective material properties in terms of process parameters such as build direction, layer height, road width or extrusion rate. Various infill patterns may be parameterized and ranked in term of their effective material properties, allowing their systematic (re)use in creating components with guaranteed mechanical performance.

\subsection{Extensions and open issues}

The proposed two-stage approach to estimating effective material properties is not limited to linear elasticity or to FDM printing. In principle, similar effective mesoscale geometry-material models of printed structures, as well as their subsequent homogenization, may be employed with other additive manufacturing process, such as Selective Laser Sintering, Stereolithography, and Inkjet printing. Other effective material properties of $3 \mathrm{D}$ printed structures may be modeled and predicted within the proposed 
computational framework. As observed in [46], similar homogenization technique applies to thermal and electrical conductivity, dielectric constant, permeability, and other material properties that share the same general form as the linear elasticity tensor.

Numerical properties of the proposed homogenization method deserve further investigation. For example, the behavior of the preconditioner $\delta C$ is not fully understood, and the condition number of the linear system has not yet been studied. Furthermore, homogenization process involves discretization errors in the implicit representation of printed roads, requires setting tolerances for convergence, and introduces discretization error of the Green's operator. Among these sources of errors, the last one in particular deserves further study. Since the Green's operator is derived and evaluated in the frequency domain, higher frequencies are lost at a low sampling resolution. Figure 9 plots the effective Young's modulus (of the same structure) at different sampling resolutions. It suggests that a minimum number of frequencies may be required for an accurate evaluation of the Green's operator. This may become particularly important when homogenizing FDM part with a small number of layers.

\section{Acknowledgements}

This research was supported by NSF grants CMMI1344205 and CMMI-1361862 and the National Institute of Standards and Technology. The authors thank Dr. John Michopoulos and Dr. John Steuben for providing the Gcodes of the specimens and properties of the filament. The responsibility for errors and omissions lies solely with the authors.

\section{Appendix A. Green's function for elasticity}

The elastic Green's function $G_{i j}\left(x, x^{\prime}\right)$ is defined as the displacement in the $i$-direction at $x$ due to a point force in the $j$-direction at $x^{\prime}$. It is the solution $u_{i}(x)$ of Equation (5) when the body force $b_{j}$ is a delta function, i.e. $b_{k}(x)=\delta\left(x-x^{\prime}\right) \delta_{j k}$ The Equation (5) and the symmetry of strain tensor directly gives

$$
\left[C_{i j k l} u_{k, l}\right]_{, j}+b_{i}=0
$$

Consider a constant point force $F$ acting at $x^{\prime}$ in $\Omega$, the displacement field caused by $F$ is

$$
u_{k}(x)=G_{k p}\left(x, x^{\prime}\right) F_{p} .
$$

The displacement gradient and stress field are

$$
\begin{aligned}
u_{k, l}(x) & =G_{k p, l}\left(x, x^{\prime}\right) F_{p} \\
\sigma_{i j}(x) & =C_{i j k l}(x) G_{k p, l}\left(x, x^{\prime}\right) F_{p} .
\end{aligned}
$$

If force $F$ is acting on point $x^{\prime} \in \Omega$, then $F$ must be balanced by the tractions on the boundary $\partial \Omega$ :

$$
\begin{gathered}
F_{i}+\int_{\partial \Omega} \sigma_{i j}(x) n_{j}(x) \mathrm{d} S=0 \\
F_{i}+\int_{\partial \Omega} C_{i j k l}(x) G_{k p, l}\left(x, x^{\prime}\right) n_{j}(x) F_{p} \mathrm{~d} x=0 .
\end{gathered}
$$

By divergence theorem

$$
F_{i}+\int_{\Omega}\left[C_{i j k l}(x) G_{k p, l}\left(x, x^{\prime}\right)\right]_{, j} F_{p} \mathrm{~d} x=0 .
$$

$F_{i}$ can be moved into the volume integral by the Dirac delta function

$$
\int_{\Omega}\left\{\left[C_{i j k l}(x) G_{k p, l}\left(x, x^{\prime}\right)\right]_{, j} F_{p}+F_{i} \delta\left(x-x^{\prime}\right)\right\} \mathrm{d} x=0 .
$$

Factoring $F_{p}$ out using Kronecker delta function

$$
\int_{\Omega}\left\{\left[C_{i j k l}(x) G_{k p, l j}\left(x, x^{\prime}\right)\right]_{, j}+\delta_{i p} \delta\left(x-x^{\prime}\right)\right\} F_{p} \mathrm{~d} x=0 .
$$

Since this holds for any arbitrary $\Omega$ and any arbitrary constant point force $F$, it must holds for every point:

$$
\left[C_{i j k l}(x) G_{k p, l}\left(x, x^{\prime}\right)\right]_{, j}+\delta_{i p} \delta\left(x-x^{\prime}\right)=0 .
$$

Since Equation (A.1) is linear in $b$, for arbitrary $b$, by superposition:

$$
u_{k}(x)=\int_{\Omega} G_{k p}\left(x, x^{\prime}\right) b_{p}\left(x^{\prime}\right) \mathrm{d} x^{\prime} .
$$

For an infinite and uniform material, $C_{i j k l}$ is constant and $G\left(x, x^{\prime}\right)=G\left(x-x^{\prime}\right)=G(x)$ by setting $x^{\prime}=0$. Then Equation (A.8) becomes:

$$
C_{i j k l} G_{k p, j l}(x)+\delta_{i p} \delta(x)=0 .
$$

Let the forward and inverse Fourier transform of $G(x)$ be

$$
\begin{aligned}
\hat{G}_{i j}(\xi) & =\int_{-\infty}^{\infty} G_{i j}(x) e^{-i \xi x} \mathrm{~d} x \\
G_{i j}(x) & =\int_{-\infty}^{\infty} \hat{G}_{i j}(\xi) e^{i \xi x} \mathrm{~d} \xi
\end{aligned}
$$

Note that index $i$ should not be confused with complex number $i=\sqrt{-1}$. With $\delta(x)=\int_{-\infty}^{\infty} e^{i \xi x} \mathrm{~d} \xi$, substitute Equation (A.11) into Equation (A.10):

$$
\int_{-\infty}^{\infty}\left[i^{2} \xi_{j} \xi_{l} C_{i j k l} \hat{G}_{k p}(\xi)+\delta_{i p}\right] e^{i \xi x} \mathrm{~d} \xi=0 .
$$

For this to be true at all $x$, we must have:

$$
\begin{array}{r}
-\xi_{j} \xi_{l} C_{i j k l} \hat{G}_{k p}(\xi)+\delta_{i p}=0 \\
\hat{G}_{k i}(\xi)=\left(C_{i j k l} \xi_{j} \xi_{l}\right)^{-1}
\end{array}
$$


From Equation 7, the Green's operator is the second order derivative of the Green's function:

$\Gamma_{m n p q}=-\frac{1}{4}\left[\frac{\partial^{2} G_{m p}}{\partial x_{n} \partial x_{q}}+\frac{\partial^{2} G_{n p}}{\partial x_{m} \partial x_{q}}+\frac{\partial^{2} G_{m q}}{\partial x_{n} \partial x_{p}}+\frac{\partial^{2} G_{n q}}{\partial x_{m} \partial x_{p}}\right]$.

In frequency domain:

$$
\begin{array}{r}
\hat{\Gamma}_{m n p q}=\frac{1}{4}\left[\left(C_{p j m l} \xi_{j} \xi_{l}\right)^{-1} \xi_{n} \xi_{q}+\left(C_{p j n l} \xi_{j} \xi_{l}\right)^{-1} \xi_{m} \xi_{q}+\right. \\
\left.\left(C_{q j m l} \xi_{j} \xi_{l}\right)^{-1} \xi_{n} \xi_{p}+\left(C_{q j n l} \xi_{j} \xi_{l}\right)^{-1} \xi_{m} \xi_{p}\right] .
\end{array}
$$

For isotropic material with Lame coefficients $\lambda$ and $\mu$ :

$$
\begin{aligned}
C_{i j k l}= & \lambda \delta_{i j} \delta_{k l}+\mu\left(\delta_{i k} \delta_{j l}+\delta_{i l} \delta_{j k}\right), \\
C_{i k j l} \xi_{k} \xi_{l}= & (\lambda+\mu) \xi_{i} \xi_{j}+\mu|\xi|^{2} \delta_{i j} \\
\hat{\Gamma}_{i j k l}= & \frac{1}{4 \mu|\xi|^{2}}\left(\delta_{i k} \xi_{l} \xi_{j}+\delta_{i l} \xi_{k} \xi_{j}+\delta_{j k} \xi_{l} \xi_{i}+\delta_{l j} \xi_{i} \xi_{k}\right) \\
& -\frac{\lambda+\mu}{\mu(\lambda+2 \mu)} \frac{\xi_{i} \xi_{j} \xi_{k} \xi_{l}}{|\xi|^{4}}
\end{aligned}
$$

\section{Appendix B. Lippmann-Schwinger equation}

Substituting Equation (6) into Equation (5) gives

$$
\left(C_{i j k l}^{0} u_{k, l}\right)_{, j}+\tau_{i j, j}+b_{i}=0 .
$$

Since equations (B.1) is linear, its solution can be separated as $u=u^{0}+u^{1}$, where $u^{0}$ is the solution of the real body force $b$ and $u^{1}$ is the solution of additional body force $\operatorname{div} \tau$ :

$$
\begin{aligned}
\left(C_{i j k l}^{0} u_{k, l}^{0}\right)_{, j}+b_{i} & =0, \\
\left(C_{i j k l}^{0} u_{k, l}^{1}\right)_{, j}+\tau_{i j, j} & =0 .
\end{aligned}
$$

Introducing the Green's function (Equation A.8) into Equation (B.3) for the comparison material, the stress polarization tensor $\tau$ becomes the "body force", from Equation (A.9)

$$
u_{i}^{1}(x)=\int_{\Omega} G_{i p}\left(x, x^{\prime}\right) \tau_{p q, q}\left(x^{\prime}\right) \mathrm{d} x^{\prime},
$$

by integrating by parts and combining with $u^{0}$

$$
u_{i}(x)=u_{i}^{0}(x)-\int_{\Omega} \frac{\partial G_{i p}\left(x, x^{\prime}\right)}{\partial x_{q}^{\prime}} \tau_{p q}\left(x^{\prime}\right) \mathrm{d} x^{\prime},
$$

by differentiation and the symmetry of strain tensor

$$
\epsilon_{i j}(x)=\epsilon_{i j}^{0}(x)-\int_{\Omega} \Gamma_{i j p q}\left(x, x^{\prime}\right) \tau_{p q}\left(x^{\prime}\right) \mathrm{d} x^{\prime},
$$

where

$$
\Gamma_{i j p q}\left(x, x^{\prime}\right)=\left.\frac{\partial^{2} G_{i p}\left(x, x^{\prime}\right)}{\partial x_{j} \partial x_{q}^{\prime}}\right|_{(i j),(p q)} .
$$

The bracketed subscripts imply symmetry, which is the results of symmetries in the elasticity tensor. Let $\delta C=C-C^{0}$, and substitute in Equation (6), Equation (B.6) becomes:

$$
\epsilon(x)=-\int_{\Omega} \Gamma\left(x, x^{\prime}\right) \delta C\left(x^{\prime}\right) \epsilon\left(x^{\prime}\right) \mathrm{d} x^{\prime}+\epsilon^{0}(x),
$$

This derivation follows the classical results of Korringa [25] and Kroner [27].

\section{Appendix C. Converting the integral equation into linear equations}

Substitute Equation (11) into Equation (7):

$$
\begin{aligned}
\epsilon(x) & =-\int_{\Omega}\left[\sum_{j=1}^{N} u_{j}(x) v_{j}\left(x^{\prime}\right)\right] \epsilon\left(x^{\prime}\right) \mathrm{d} x^{\prime}-\epsilon^{0}(x) \\
& =-\sum_{j=1}^{N}\left[u_{j}(x) \int_{\Omega} v_{j}\left(x^{\prime}\right) \epsilon\left(x^{\prime}\right) \mathrm{d} x^{\prime}\right]+\epsilon^{0}(x),
\end{aligned}
$$

Since $\int_{\Omega} v_{j}\left(x^{\prime}\right) \epsilon\left(x^{\prime}\right) \mathrm{d} x^{\prime}$ is the sampling of continuous $\epsilon$ over $\Omega$, let

$$
\int_{\Omega} v_{j}\left(x^{\prime}\right) \epsilon\left(x^{\prime}\right) \mathrm{d} x^{\prime}=\epsilon_{j} .
$$

Substitute Equation (C.2) into Equation (C.1):

$$
\epsilon(x)=-\sum_{j=1}^{N} u_{j}(x) \epsilon_{j}+\epsilon^{0}(x) .
$$

Substitute Equation (C.3) back into Equation (C.2), we have

$$
\begin{aligned}
\epsilon_{i} & =-\int_{\Omega} v_{i}\left(x^{\prime}\right)\left[-\sum_{j=1}^{N} u_{j}\left(x^{\prime}\right) \epsilon_{j}+\epsilon^{0}\left(x^{\prime}\right)\right] \mathrm{d} x^{\prime} \\
& =-\sum_{j=1}^{N} \epsilon_{j} \int_{\Omega} v_{i}\left(x^{\prime}\right) u_{j}\left(x^{\prime}\right) \mathrm{d} x^{\prime}+\int_{\Omega} v_{i}\left(x^{\prime}\right) \epsilon^{0}\left(x^{\prime}\right) \mathrm{d} x^{\prime} \\
& =-\sum_{j=1}^{N} \Gamma(i, j) \delta C(j) \epsilon_{j}+\epsilon_{i}^{0} \\
\epsilon_{i}^{0} & =\left[\delta_{i j}+\sum_{j=1}^{N} \Gamma(i, j) \delta C(j)\right] \epsilon_{j}=\left[\delta_{i j}+\sum_{j=1}^{N} \Gamma_{i j} \delta C_{j}\right] \epsilon_{j} .
\end{aligned}
$$

\section{Appendix D. Positive definiteness}

This section follows the proof given in [42], which requires virtual work equality:

$$
\sigma_{1} \epsilon_{2}=0
$$

for any divergence free stress $\sigma_{1}$ and strain $\epsilon_{2}$ derived from a displacement that is 0 over the boundary. First, we show that $\Gamma$ is positive definite. Since

$$
\tau_{1} \Gamma \tau_{2}=-\tau_{1} \epsilon_{2}=-\delta C \epsilon_{1} \epsilon_{2}=\epsilon_{1} C_{0} \epsilon_{2}
$$

$\Gamma$ is seen to be positive definite after setting $\epsilon_{1}=\epsilon_{2}$, as $C_{0}$ is positive definite.

When $\delta C$ is positive definite, the positive definiteness of $B$ is shown in text. Here we show the case when $\delta C$ is negative definite. It is convenient to introduce strain polarization tensor $\eta$ :

$$
\tau=C_{0} \eta, \quad \eta=S_{0} \tau=S_{0}\left(C-C_{0}\right) \epsilon=S_{0} \sigma-\epsilon
$$


Using Equation (D.1) again,

$$
\begin{aligned}
\tau_{1} \Gamma \tau_{2} & =-\tau_{1} \epsilon_{2}=\eta_{1} C_{0}\left(\eta_{2}-S_{0} \sigma_{2}\right) \\
& =\eta_{1} C_{0} \eta_{2}-\eta_{1} \sigma_{2}=\eta_{1} C_{0} \eta_{2}-\sigma_{1} S_{0} \sigma_{2}
\end{aligned}
$$

Since $\left(C-C_{0}\right)^{-1} C_{0}=S_{0}\left(S_{0}-S\right)^{-1}-I$,

$$
\begin{aligned}
\tau\left(C-C_{0}\right)^{-1} \tau & =\eta L_{0}\left(C-C_{0}\right)^{-1} L_{0} \eta \\
& =\eta\left(S_{0}-S\right)^{-1} \eta-\eta C_{0} \eta
\end{aligned}
$$

Then $\delta C^{-1}+\Gamma=\eta\left(S_{0}-S\right)^{-1} \eta-\sigma_{1} S_{0} \sigma_{2}$ is negative definite when $\delta C$ is negative definite.

\section{References}

[1] D. Ahn, J.-H. Kweon, S. Kwon, J. Song, and S. Lee, "Representation of surface roughness in fused deposition modeling," $J$. Mater. Process. Technol., vol. 209, no. 15-16, pp. 5593-5600, 2009.

[2] A. Boschetto, V. Giordano, and F. Veniali, "Modelling micro geometrical profiles in fused deposition process," Int. J. Adv. Manuf. Technol., vol. 61, no. 9-12, pp. 945-956, 2011.

[3] A. K. Sood, R. K. Ohdar, and S. S. Mahapatra, "Improving dimensional accuracy of Fused Deposition Modelling processed part using grey Taguchi method," Mater. Des., vol. 30, no. 10, pp. 4243-4252, 2009.

[4] P.-H. Lee, H. Chung, S. W. Lee, J. Yoo, and J. Ko, "Review: Dimensional Accuracy in Additive Manufacturing Processes," in Vol. 1 Mater. Micro Nano Technol. Prop. Appl. Syst. Sustain. Manuf., p. V001T04A045, 2014.

[5] A. Telea and A. Jalba, "Voxel-based assessment of printability of 3D shapes," Lect. Notes Comput. Sci. (including Subser. Lect. Notes Artif. Intell. Lect. Notes Bioinformatics), vol. 6671 LNCS, pp. 393-404, 2011.

[6] S. Nelaturi and V. Shapiro, "Representation and analysis of additively manufactured parts," Computer-Aided Design, vol. 6768, pp. 13-23, 2015.

[7] "ASTM D3039 / D3039M-14, Standard Test Method for Tensile Properties of Polymer Matrix Composite Materials,", 2014.

[8] S.-H. Ahn, M. Montero, D. Odell, S. Roundy, and P. K. Wright, "Anisotropic material properties of fused deposition modeling ABS," Rapid Prototyp. J., vol. 8, no. 4, pp. 248-257, 2002.

[9] M. Dawoud, I. Taha, and S. J. Ebeid, "Mechanical behaviour of ABS: An experimental study using FDM and injection moulding techniques," J. Manuf. Process., vol. 21, pp. 39-45, 2016.

[10] J. Steuben, A. Iliopoulos, and J. Michopoulos, "Implicit Slicing for Functionally Tailored Additive Manufacturing," ComputerAided Desingn, 2015. submitted for publication.

[11] A. Ranellucci, "Slic3r: Gcode generator for 3D printers," 2015.

[12] D. Ahn, J.-H. Kweon, S. Kwon, J. Song, and S. Lee, "Representation of surface roughness in fused deposition modeling," $J$. Mater. Process. Technol., vol. 209, no. 15-16, pp. 5593-5600, 2009.

[13] S. Nelaturi, W. Kim, and T. Kurtoglu, "Manufacturability Feedback and Model Correction for Additive Manufacturing," $J$. Manuf. Sci. Eng., vol. 137, no. 2, p. 21015, 2015.

[14] B. McCarthy, "Characterization of geometric deviations in FDM," Master's thesis, University of Wisconsin - Madison, 2015.

[15] C. Casavola, A. Cazzato, V. Moramarco, and C. Pappalettere, "Orthotropic mechanical properties of fused deposition modelling parts described by classical laminate theory," Mater. Des., vol. 90, pp. 453-458, 2016.

[16] C. W. Ziemian, M. M. Sharma, and S. N. Ziemian, "Anisotropic Mechanical Properties of ABS Parts Fabricated by Fused Deposition Modelling," Mech. Eng., pp. 159-180, 2012.

[17] J. Kotlinski, "Mechanical properties of commercial rapid prototyping materials," Rapid Prototyp. J., vol. 20, no. 6, pp. 499-510, 2014 .
[18] S. J. Hollister and N. Kikuehi, "A comparison of homogenization and standard mechanics analyses for periodic porous composites," Comput. Mech., pp. 73-95, 1992.

[19] T. Kanit, S. Forest, I. Galliet, V. Mounoury, and D. Jeulin, "Determination of the size of the representative volume element for random composites: Statistical and numerical approach," Int. J. Solids Struct., vol. 40, no. 13-14, pp. 3647-3679, 2003.

[20] K. Terada, M. Hori, T. Kyoya, and N. Kikuchi, "Simulation of the multi-scale convergence in computational homogenization approaches," Int. J. Solids Struct., vol. 37, no. 16, pp. 2285-2311, 2000

[21] J. Panetta, Q. Zhou, L. Malomo, N. Pietroni, P. Cignoni, and D. Zorin, "Elastic textures for additive fabrication," ACM Trans. Graph., vol. 34, no. 4, pp. 135:1-135:12, 2015.

[22] C. Schumacher and B. Bickel, "Microstructures to Control Elasticity in 3D Printing," ACM Trans. Graph. (ACM SIGGRAPH 2015), vol. 34, no. 4, 2015.

[23] E. Andreassen, B. S. Lazarov, and O. Sigmund, "Design of manufacturable 3D extremal elastic microstructure," Mech. Mater., vol. 69 , no. 1, pp. 1-10, 2014.

[24] C.-T. Wu and R. L. McCullough, "Constitutive relationships for heterogeneous materials(elastic properties of polycrystals and composite materials)," Dev. Compos. Mater. 78-38276 16-24) London, Appl. Sci. Publ. Ltd., pp. 119-187, 1977.

[25] J. Korringa, "Theory of elastic constants of heterogeneous media," J. Math. Phys., vol. 14, no. 4, pp. 509-513, 1973.

[26] D. R. S. Talbot and J. R. Willis, "Bounds and self-consistent estimates for the overall properties of nonlinear composites," IMA J. Appl. Math. (Institute Math. Its Appl., vol. 39, no. 3, pp. 215-240, 1987.

[27] E. Kröner, "Bounds for effective elastic moduli of disordered materials," J. Mech. Phys. Solids, vol. 25, no. 2, pp. 137-155, 1977.

[28] W. J. Drugan and J. R. Willis, "A micromechanics-based nonlocal constitutive equation and estimates of representative volume element size for elastic composites," J. Mech. Phys. Solids, vol. 44, no. 4, pp. 497-524, 1996.

[29] H. Moulinec and P. Suquet, "A numerical method for computing the overall response of nonlinear composites with complex microstructure," Comput. Methods Appl. Mech. Eng., vol. 157, no. $1-2$, pp. $69-94,1998$

[30] S. Brisard and L. Dormieux, "Combining Galerkin approximation techniques with the principle of Hashin and Shtrikman to derive a new FFT-based numerical method for the homogenization of composites," Comput. Methods Appl. Mech. Eng., vol. $217-220$, pp. 197-212, 2012.

[31] J. C. Michel, H. Moulinec, and P. Suquet, "A computational scheme for linear and non-linear composites with arbitrary phase contrast," in 5th U.S. Natl. Congr. Comput. Mech. 4-6 Aug. 1999, vol. 52, pp. 139-160, 2001.

[32] V. Vinogradov and G. Milton, "An accelerated FFT algorithm for thermoelastic and non-linear composites," Int. J. Numer. Methods Eng., vol. 76, no. 11, pp. 1678-1695, 2008.

[33] S. Brisard and L. Dormieux, "FFT-based methods for the mechanics of composites: A general variational framework," Comput. Mater. Sci., vol. 49, no. 3, pp. 663-671, 2010.

[34] J. Zeman, J. Vondejc, J. Novák, and I. Marek, "Accelerating a FFT-based solver for numerical homogenization of periodic media by conjugate gradients," J. Comput. Phys., vol. 229, no. 21 , pp. 8065-8071, 2010.

[35] J. Vondrejc, J. Zeman, and I. Marek, "An FFT-based Galerkin method for homogenization of periodic media," Comput. Math with Appl., vol. 68, no. 3, pp. 156-173, 2014

[36] S. Moser, "Mesostructured Cellular Materials Generator." http: //www.thingiverse.com/thing: 304177, Date accessed: 2016-0202 .

[37] C. Bellehumeur, L. Li, Q. Sun, and P. Gu, "Modeling of Bond Formation Between Polymer Filaments in the Fused Deposition Modeling Process," J. Manuf. Process., vol. 6, no. 2, pp. 170-178, 2004.

[38] Q. Sun, G. Rizvi, C. Bellehumeur, and P. Gu, "Effect of process- 
ing conditions on the bonding quality of FDM polymer filaments," Rapid Prototyp. J., vol. 14, no. 2, pp. 72-80, 2008.

[39] L. E. Malvern, Introduction to the mechanics of a continuous medium. Prentice-Hall series in engineering of the physical sciences, Prentice-Hall, 1969.

[40] A. C. Pipkin, A course on integral equations. No. 9, Springer Science \& Business Media, 1991.

[41] J. R. Shewchuk, "An Introduction to the Conjugate Gradient Method Without the Agonizing Pain," Science (80-. )., vol. 49, no. CS-94-125, p. 64, 1994.

[42] J. Willis, "Bounds and self-consistent estimates for the overall properties of anisotropic composites," J. Mech. Phys. Solids, vol. 25, pp. 185-202, jun 1977.

[43] K. Suresh, "Efficient Microstructural Design for Additive Manufacturing," in ASME 2014 Int. Des. Eng. Tech. Conf. Comput. Inf. Eng. Conf., pp. V01AT02A045-V01AT02A045, American Society of Mechanical Engineers, 2014.

[44] M. S. Mamadapur, "Constitutive Modeling of Fused Deposition Modeling Acrylonitrile Butadiene Styrene (ABS)," Master's thesis, Texas A \& M University, 2007.

[45] C. Hoffmann, V. Shapiro, and V. Srinivasan, "Geometric interoperability via queries," Computer-Aided Design, vol. 46, no. 1, pp. 148-159, 2014.

[46] S. Torquato, Random heterogeneous materials: microstructure and macroscopic properties. Interdisciplinary applied mathematics: Mechanics and materials, Springer, 2002. 